\title{
ВИМІРЮВАЛЬНІ СИСТЕМИ
}

УДК 681.242 .389

\section{ЗАСТОСУВАННЯ ЕЛЕМЕНТІВ ТЕОРІЇ КІНЦЕВИХ АВТОМАТІВ ДЛЯ ДОСЛІДЖЕНЬ ДИНАМІЧНИХ ВЛАСТИВОСТЕЙ ОБЧИСЛЮВАЛЬНИХ КОМПОНЕНТІВ ВИМІРЮВАЛЬНИХ СИСТЕМ}

(C) Кричевець Олександр, 2017

Державне підприємство “Науково-дослідний інститут метрології вимірювальних та управляючих систем”, вул. М. Кривоноса, 679008, Львів, Україна

Розглянуто динамічні властивості обчислювальних компонентів вимірювальних систем на базі теорії елементарних кінцевих автоматів. Показано, це динамічні властивості обчислювальних компонентів у представленні елементарного автомата Мілі виникають переважно внаслідок змін вхідних сигналів $x$ (t). Для обчислювальних компонентів у представленні елементарного автомата Мура динамічні властивості залежать від метрологічного стану компонентів. Подано результати розрахунків динамічних характеристик обчислювальних компонентів, які використовують типові обчислювальні алгоритми. Ключові слова: обчислювальний компонент, автомат Мілі, автомат Мура, динамічні характеристики.

Рассмотрены динамические свойства вычислительных компонентов измерительных систем на базе теории элементарных конечных автоматов. Показано, что динамические свойства вычислительных компонентов в

представлении элементарного автомата Мили возникают преимущественно в результате изменений входных сигналов $\boldsymbol{x}(\boldsymbol{t})$. Для вычислительных компонентов в представлении элементарного автомата Мура динамические свойства зависят от метрологического состояния компонентов. Представлены результаты расчетов динамических характеристик вычислительных компонентов, которые используют типовые вычислительные алгоритмы.

Ключевые слова: вычислительный компонент, автомат Мили, автомат Мура, динамические характеристики.

The results of research dynamic properties of computing components of measuring systems is considered. It is shown that the dynamic properties of computing components in the presentation Mealy machine arising due to the dynamic changes in input signals $x(t)$. For the computing components to present Moore dynamic properties are determined mainly dynamic changes of metrological conditions computing components.

A distinctive feature of Mealy machines is that their output signals depend on the state of the machine, and the value of the input signal. In Moore machine outputs $y(t)$ at each discrete time $t$ is uniquely determined by the state machine at the same time and do not depend on the input signal. Hence, if the input mealy machine that keeps the same state will change the input of its value and in time, the output signal will change according to the algorithm of the machine. In the Moore machine this happens, change the output signal is possible only after the change in status.

Therefore, when you receive repeated the same input signal at the outputs Moore machine can form various signals. In Moore machines forming the output signal takes time, and is usually divided into cycles. In each cycle, depending on the input signal and state machine output signal is formed and a new state. Thus, we can say that the output signal Moore automaton depends on the sequence of inputs. This causes the output depending on the time of admission to the input signal machine.

Hence, one should note the existence of dynamic properties of the machine during operation.

So the dynamic properties of computing component in the presentation Mealy machine arising due to the dynamic changes in the time of the input signals $x(t)$. For the computing component to present Moore machine dynamic properties are determined mainly dynamic changes of metrological conditions of computing component. 
Then the dynamic properties of computing component in the presentation Mealy machine determined dynamic characteristic - the dependence of error of the results of numerical measurement conversion on the values of the error signal input at the time, and for the computing component to present Moore machine-dependent error numerical measuring conversion of options approach, which, in fact, responsible for change computing component metrological conditions at the time.

The first type conversion is typical in the implementation of computational algorithms for the calculation formula, and the other type - in the implementation of computational algorithms for approximate calculation.

A computational experiments with different types of computer algorithms are presented. For the first type conversion formula used for linear dependencies. For the second-iterative procedures and numerical integration.

Key words: computing component, Mealy machine, Moore machine, dynamic characteristic.

Вступ. Обчислювальні компоненти (ОК) вимірювальних систем, що являють собою числові вимірювальні перетворювачі, мають особливі специфічні властивості, які відрізняють їх від апаратних вимірювальних перетворювачів. Базовою відмінністю функціональних властивостей ОК від ЗВТ $є$ те, що ОК реалізують детерміновані процедури над дискретними масивами даних, звідси ОК доцільно описати моделлю кінцевого автомата. Модель кінцевого автомата задається математичною схемою, яка характеризується шістьма елементами: кінцевою множиною $X$ вхідних даних (вхідний алфавіт), кінцевою множиною $Y$ вихідних даних (вихідний алфавіт), кінцевою множиною $Z$ внутрішніх станів (алфавіт станів), початковим станом $Z_{0}$, перехідною функцією $\varphi(z, x)$ та функцією виходів $\psi(z, x)$. У кожний момент $t=0,1,2$ дискретного часу автомат перебуває у певному стані $z(t)$ з множини $Z$ станів автомата, в початковий момент часу $t=0$ він завжди буде у початковому стані $z(0)=z_{0}$. На момент $t$, будучи у стані $z(t)$, автомат сприймає на вході сигнал $x(t)$ і видає на виході сигнал $y(t)=\psi[z(t), x(t)]$, переходячи у стан $z(t+1)=\varphi[z(t), x(t)]$. У поданні кінцевого автомата узагальнена модель ОК виглядатиме так:

- стан на момент часу $t-Z(t)$; Множина вхідних даних $-X(t)+\Delta X(t ;)$

- функція виходів - $Y(t)+\Delta Y(t)=\psi[Z(t)$, $(X(t)+\Delta X(t))]$;

- перехідна функція виду:

$$
\varphi[Z(t),(X(t)+\Delta X(t))] .
$$

де $\Delta X(t)$ - похибка вхідних даних; $\Delta Y(t)$ - похибка вихідних даних.

Зі співвідношень (1) випливає, що ОК реалізують метрологічну функцію, а саме: перетворення похибок масивів вхідних даних $\Delta X(t) \rightarrow \Delta Y(t)$, причому кожне значення як похибок вхідних даних, так і вихідних даних приписується конкретному фіксованому часу i відповідному метрологічному стану ОК. Кількісно метрологічний стан ОК визначається значеннями коефіцієнта перетворення похибок вхідних даних $f$ у певному такті, в якому визначається результат вимірювання з похибкою $\Delta Y(t)$. Попередні дослідження ОК вимірювальних систем показали доцільність використання елементів теорії кінцевих автоматів для оцінки їхніх метрологічних властивостей [1-5]. Поперше, доведено, що метрологічні характеристики ОК детерміновані, по-друге, визначено важливу характеристику ОК - коефіцієнт перетворення, яка визначає метрологічний стан ОК під час проведення обчислень, по-третє, виявлено залежність коефіцієнтів перетворення ОК від ймовірності виникнення обчислювальних схем під час функціонування "гнучких" ОК. Водночас не досліджено динамічних властивостей ОК, які відіграють істотну роль у виникненні додаткових похибок вимірювальних систем.

Метою роботи $є$ дослідження динамічних характеристик ОК з використанням елементів теорії кінцевих автоматів.

Теоретичні основи. Зазвичай автомати прийнято розділяти на два класи - елементарні автомати Мілі й автомати Мура [6, 7]. Автомат Мілі можна описати рівнянням (2). Модель автомата Мура описана рівнянням (3):

$$
\begin{gathered}
a(t+1)=\delta[a(t), x(t)], y(t)=\lambda[a(t), \\
x(t)] \quad(t=0,1,2, \ldots) . \\
a(t+1)=\delta[a(t), x(t)], y(t)=\lambda(a(t)), \\
(t=0,1,2, \ldots) .
\end{gathered}
$$

де $a(t)$ - значення стану автомата; $x(t)$ - вхідний сигнал; $\delta(a(t), x(t))$ - функція переходів автомата; $\lambda(a(t)$, $x(t))$ - функція виходів автомата. 
Відмінною особливістю автоматів Мілі є те, що їхні вихідні сигнали залежать і від стану автомата, і від значення вхідного сигналу. Автомат Мілі описується двома матрицями: матрицею переходів (табл. 1) і матрицею виходів (табл. 2).

Таблиия 1

\section{Матриця переходів}

Table 1

\section{Transition matrix}

\begin{tabular}{|c|c|c|c|}
\hline $\mathrm{x}_{\mathrm{j}} \backslash \mathrm{a}_{\mathrm{i}}$ & $\mathrm{a}_{\mathrm{o}}$ & $\cdots$ & $\mathrm{a}_{\mathrm{m}}$ \\
\hline $\mathrm{x}_{1}$ & $\delta\left(\mathrm{a}_{\mathrm{o}}, \mathrm{x}_{1}\right)$ & $\cdots$ & $\delta\left(\mathrm{a}_{\mathrm{m}}, \mathrm{x}_{1}\right)$ \\
\hline$\cdots$ & $\cdots$ & $\cdots$ & $\cdots$ \\
\hline $\mathrm{x}_{\mathrm{m}}$ & $\delta\left(\mathrm{a}_{\mathrm{o}}, \mathrm{x}_{\mathrm{m}}\right)$ & $\cdots$ & $\delta\left(\mathrm{a}_{\mathrm{m}}, \mathrm{x}_{\mathrm{m}}\right)$ \\
\hline
\end{tabular}

Таблиия 2

\section{Матриця виходів}

Table 2

\section{Output matrix}

\begin{tabular}{|c|c|c|c|}
\hline $\mathrm{x}_{\mathrm{j}} \backslash \mathrm{a}_{\mathrm{i}}$ & $\mathrm{a}_{\mathrm{o}}$ & $\cdots$ & $\mathrm{a}_{\mathrm{m}}$ \\
\hline $\mathrm{x}_{1}$ & $\lambda\left(\mathrm{a}_{\mathrm{o}}, \mathrm{x}_{1}\right)$ & $\cdots$ & $\lambda\left(\mathrm{a}_{\mathrm{m}}, \mathrm{x}_{1}\right)$ \\
\hline$\cdots$ & $\ldots$ & $\cdots$ & $\cdots$ \\
\hline $\mathrm{x}_{\mathrm{m}}$ & $\lambda\left(\mathrm{a}_{\mathrm{o}}, \mathrm{x}_{\mathrm{m}}\right)$ & $\cdots$ & $\lambda\left(\mathrm{a}_{\mathrm{m}}, \mathrm{x}_{\mathrm{m}}\right)$ \\
\hline
\end{tabular}

Рядки цих матриць відповідають вхідним сигналам $x(t)$, а стовпці - станам. На перетині стовпця $a_{i}$ і рядка $x_{j}$ У матриці переходів ставиться стан $a_{j}=\delta\left[a_{i}, x_{j}\right]$, в який автомат перейде зі стану $a_{i}$ під впливом сигналу $x_{j}$; а в матриці виходів - відповідає цьому переходу вихідний сигнал $y=\lambda\left[a_{i}, x_{j}\right]$. В автоматах Мура вихідні сигнали $y$ $(t)$ в кожен дискретний момент часу $t$ однозначно визначаються станом автомата в той самий момент часу і не залежать від значення вхідного сигналу. Щоб задати його, потрібна тільки т. зв. зазначена матриця переходів автомата Мура (табл. 3).

Таблиияя 3

Зазначена матриця переходів

Table 3

Set transition matrix

\begin{tabular}{|c|c|c|c|}
\hline $\mathrm{y}$ & $\lambda\left(\mathrm{a}_{0}\right)$ & $\cdots$ & $\lambda\left(\mathrm{a}_{\mathrm{n}}\right)$ \\
\hline $\mathrm{x}_{\mathrm{j}} \mathrm{a}_{\mathrm{c}}$ & $\mathrm{a}_{0}$ & $\cdots$ & $\mathrm{a}_{\mathrm{n}}$ \\
\hline $\mathrm{x}_{1}$ & $\delta\left(\mathrm{a}_{0}, \mathrm{x}_{1}\right)$ & $\cdots$ & $\delta\left(\mathrm{a}_{\mathrm{n}}, \mathrm{x}_{1}\right)$ \\
\hline$\ldots$ & $\ldots$ & $\cdots$ & $\cdots$ \\
\hline $\mathrm{x}_{\mathrm{m}}$ & $\delta\left(\mathrm{a}_{0}, \mathrm{x}_{\mathrm{m}}\right)$ & $\cdots$ & $\delta\left(\mathrm{a}_{\mathrm{n}}, \mathrm{x}_{\mathrm{m}}\right)$ \\
\hline
\end{tabular}

Звідси, якщо на вході автомата Мілі, що зберігає той самий стан, відбудеться зміна вхідного сигналу за його значенням і в часі, то зміниться і вихідний сигнал відповідно до алгоритму роботи автомата. В автоматі Мура цього не станеться: зміна вихідного сигналу можлива тільки після зміни стану. Тому у випадку багаторазового надходження одного i того самого вхідного сигналу на виходах автомата Мура можуть формуватися різні сигнали.

В автоматах Мура формування вихідного сигналу займає певний час, який зазвичай поділяється на такти. У кожному такті залежно від вхідного сигналу і стану автомата формується вихідний сигнал і новий стан. Отже, можна сказати, що вихідний сигнал автомата Мура залежить від послідовності вхідних сигналів. Це призводить до залежності вихідного сигналу від часу надходження сигналів на вхід автомата. Звідси можна констатувати наявність динамічних властивостей автоматів під час функціонування. Ураховуючи наведені дані, розглянемо метрологічні моделі ОК у представленні автомата Мілі та автомата Мура. Тоді матрицю переходів для ОК у представленні автомата Мілі подано в табл. 4, а матрицю виходів - в табл. 5.

Таблиия 4

\section{Матриця переходів ОК}

Table 4

Transition matrix of computing component

\begin{tabular}{|c|c|}
\hline $\mathrm{x}_{\mathrm{j}} \mid \mathrm{a}_{\mathrm{i}}$ & $\mathrm{a}_{\mathrm{o}}$ \\
\hline $\mathrm{x}_{1}\left(\mathrm{t}_{1}\right)_{+} \Delta \mathrm{x}_{1}\left(\mathrm{t}_{1}\right)$ & $\delta\left(\mathrm{a}_{\mathrm{o}},\left(\mathrm{x}_{1}\left(\mathrm{t}_{1}\right)_{+} \Delta \mathrm{x}_{1}\left(\mathrm{t}_{1}\right)\right)\right.$ \\
\hline$\cdots$ & $\cdots$ \\
\hline $\mathrm{x}_{\mathrm{m}}\left(\mathrm{t}_{\mathrm{m}}\right)_{+} \Delta \mathrm{x}_{\mathrm{m}}\left(\mathrm{t}_{\mathrm{m}}\right)$ & $\delta\left(\mathrm{a}_{\mathrm{o}},\left(\mathrm{x}_{\mathrm{m}}\left(\mathrm{t}_{\mathrm{m}}\right)_{+} \Delta \mathrm{x}_{\mathrm{m}}\left(\mathrm{t}_{\mathrm{m}}\right)\right)\right.$ \\
\hline
\end{tabular}

Таблиияя 5

Матриця виходів ОК

Table 5

Output matrix of computing component

\begin{tabular}{|c|c|}
\hline $\mathrm{x}_{\mathrm{j}} \backslash \mathrm{a}_{\mathrm{i}}$ & $\mathrm{a}_{\mathrm{o}}$ \\
\hline $\mathrm{x}_{1}\left(\mathrm{t}_{1}\right)_{+} \Delta \mathrm{x}_{1}\left(\mathrm{t}_{1}\right)$ & $\mathrm{y}_{1}\left(\mathrm{t}_{1}\right)_{+} \Delta \mathrm{y}_{1}\left(\mathrm{t}_{1}\right)=\lambda\left(\mathrm{a}_{\mathrm{o}}, \mathrm{x}_{1}\left(\mathrm{t}_{1}\right)\right)$ \\
\hline$\cdots$ & $\cdots$ \\
\hline $\mathrm{x}_{\mathrm{m}}\left(\mathrm{t}_{\mathrm{m}}\right)_{+} \Delta \mathrm{x}_{\mathrm{m}}\left(\mathrm{t}_{\mathrm{m}}\right)$ & $\mathrm{y}_{\mathrm{m}}\left(\mathrm{t}_{\mathrm{m}}\right)_{+} \Delta \mathrm{y}\left(\mathrm{t}_{\mathrm{m}}\right)=\lambda\left(\mathrm{a}_{\mathrm{o}}, \mathrm{x}_{\mathrm{m}}\left(\mathrm{t}_{\mathrm{m}}\right)\right)$ \\
\hline
\end{tabular}

Зазначена матриця переходів для ОК у представленні автомата Мура матиме вигляд: 


\section{Таблиия 6}

Зазначена матриця переходів ОK

Table 6

Set transition matrix of computing component

\begin{tabular}{|c|c|c|c|}
\hline $\mathrm{x}_{\mathrm{j}} \mathrm{a}_{\mathrm{c}}$ & $\mathrm{a}_{0}$ & $\cdots$ & $\mathrm{a}_{\mathrm{n}}$ \\
\hline $\mathrm{x}_{1}\left(\mathrm{t}_{1}\right)_{+} \Delta \mathrm{x}_{1}\left(\mathrm{t}_{1}\right)$ & $\delta\left(\mathrm{a}_{\mathrm{o}},\left(\mathrm{x}_{1}\left(\mathrm{t}_{1}\right)_{+} \Delta \mathrm{x}_{1}\left(\mathrm{t}_{1}\right)\right)\right.$ & $\cdots$ & $\delta\left(\mathrm{a}_{\mathrm{n}},\left(\mathrm{x}_{\mathrm{n}}\left(\mathrm{t}_{1}\right)_{+} \Delta \mathrm{x}_{\mathrm{n}}\left(\mathrm{t}_{1}\right)\right)\right.$ \\
\hline$\ldots$ & $\ldots$ & $\cdots$ & $\ldots$ \\
\hline $\mathrm{x}_{\mathrm{m}}\left(\mathrm{t}_{\mathrm{m}}\right)_{+} \Delta \mathrm{x}_{\mathrm{m}}\left(\mathrm{t}_{\mathrm{m}}\right)$ & $\delta\left(\mathrm{a}_{0},\left(\mathrm{x}_{\mathrm{m}}\left(\mathrm{t}_{\mathrm{m}}\right)_{+} \Delta \mathrm{x}_{\mathrm{m}}\left(\mathrm{t}_{\mathrm{m}}\right)\right)\right.$ & $\cdots$ & $\delta\left(\mathrm{a}_{\mathrm{n}},\left(\mathrm{x}_{\mathrm{m}}\left(\mathrm{t}_{\mathrm{m}}\right)_{+} \Delta \mathrm{x}_{\mathrm{m}}\left(\mathrm{t}_{\mathrm{m}}\right)\right)\right.$ \\
\hline $\mathrm{y}_{\mathrm{m}}\left(\mathrm{t}_{\mathrm{m}}\right)_{+} \Delta \mathrm{y}\left(\mathrm{t}_{\mathrm{m}}\right)$ & $\lambda\left(\mathrm{a}_{\mathrm{o}}, \mathrm{x}_{\mathrm{m}}\left(\mathrm{t}_{\mathrm{m}}\right)\right)$ & $\cdots$ & $\lambda\left(\mathrm{a}_{\mathrm{n}}, \mathrm{x}_{\mathrm{m}}\left(\mathrm{t}_{\mathrm{m}}\right)\right)$ \\
\hline
\end{tabular}

де $a_{0}, a_{1} \ldots a_{n}$ - значення метрологічних станів ОК [3].

Отже, динамічні властивості ОК у представленні автомата Мілі виникають за рахунок динамічних змін у часі вхідних сигналів $x(t)$. Для ОК у представленні автомата Мура динамічні властивості визначаються переважно динамікою змін метрологічних станів ОК. Тоді динамічні властивості ОК у представленні автомата Мілі визначатимуться динамічною характеристикою - залежністю похибки результатів числового вимірювального перетворення від значень похибки вхідного сигналу у часі, а для ОК у представленні автомата Мура - залежністю похибки числового вимірювального перетворення від параметрів наближення, які, власне, відповідають за зміни метрологічних станів ОК у часі. Перший тип перетворення характерний під час реалізації обчислювальних алгоритмів розрахунку за формулами, а другий тип - під час реалізації обчислювальних алгоритмів наближеного розрахунку [3]. Здійснено обчислювальні експерименти з різними типами обчислювальних алгоритмів. Для першого типу перетворення використовувалися формули розрахунку для лінійних залежностей. Для другого ітераційні процедури та процедури числового інтегрування.

Визначання динамічної характеристики обчислювального компонента, що реалізує процедуру розрахунку за формулою (лінійна залежність). Розглянемо процедуру лінеаризації результатів прямих вимірювань певної фізичної величини $x$ за формулою:

$$
y=2 x+3 .
$$

На вхід обчислювального компонента подавалося значення $x=0,1$, яке приймають за дійсне значення фізичної величини. Отримане значення $y_{0}=3.2$ фіксують. Далі формують масив $n$ "засмічених" значень
$\left\{x_{i}^{\prime}\right\}=\left\{x_{i}+\Delta x_{i}\right\}$ в діапазоні \pm 0.5 використовуючи, наприклад, генератор псевдовипадкових чисел. Інтервал значень $\Delta x_{i}$ має збігатись з інтервалом похибок результатів прямих вимірювань. Розрахунок виконують за формулою (3), отримуючи масив $n$ значень $\left\{y_{i}^{\prime}\right\}$. Формують масив похибок обчислень величини:

$$
\Delta y_{i}=\left\{y_{i}^{\prime}-y_{0}\right\}, i=1 \ldots n \text {. }
$$

Розраховують коефіцієнти перетворення ОК (функцію переходів) $f_{i}$ за формулою

$$
f_{i}=\Delta y_{i} / \Delta x_{i} p \text { за умови, що } \Delta x_{i}>0 .
$$

Отримано масив значень $\left\{f_{i}\right\}$. Результати обчислень наведено в табл. 7.

Таблиия 7

\section{Розрахунок коефіціснтів перетворення ОК}

Table 7

Calculation of computing component conversion coefficient

\begin{tabular}{|c|c|c|c|c|}
\hline$\Delta x_{i}(t)$ & $x_{I}(t)$ & $y_{i}^{\prime}(t)$ & $\Delta y_{i}(t)$ & $f_{i}$ \\
\hline$-0,05$ & 0,05 & 3,1 & $-0,1$ & 2,0 \\
\hline$-0,04$ & 0,06 & 3,12 & $-0,08$ & 2,0 \\
\hline$-0,02$ & 0,08 & 3,16 & $-0,04$ & 2,0 \\
\hline 0,01 & 0,11 & 3,22 & 0,02 & 2,0 \\
\hline 0,02 & 0,12 & 3,24 & 0,04 & 2,0 \\
\hline 0,03 & 0,13 & 3,26 & 0,06 & 2,0 \\
\hline 0,04 & 0,14 & 3,28 & 0,08 & 2,0 \\
\hline 0,05 & 0,15 & 3,30 & 0,10 & 2,0 \\
\hline
\end{tabular}

За результатами обчислень приймають значення коефіцієнта перетворення обчислювального компонента (функції переходів) у діапазоні значень похибок прямих вимірювань $f=2,0$. Це означає, що метрологічний стан ОК для цього обчислення $є$ незмінним. Використовуючи дані табл. 7, визначаємо динамічну характеристику цього ОК. Зміна вихідного сигналу $y_{i}^{\prime}=y_{0} \pm \Delta y_{i}$ відбувається за рахунок змін у похибках вхідних сигналів у часі $\Delta x_{i}(t)$. Звідси динамічна характеристика ОК $є$ залежністю $\Delta y_{i}(t)=\Delta x_{i}(t)=\varphi(t)$.

Визначання динамічних характеристик обчислювального компонента, який реалізує процедуру ітераційного розв'язування системи лінійних рівнянь методом Якобі. Розглядають систему з трьох лінійних рівнянь:

$$
\left\{\begin{array}{l}
12 x_{1}-3 x_{2}+x_{3}=9 \\
x_{1}+5 x_{2}-x_{3}=8 \\
x_{1}-x_{2}+3 x_{3}=8
\end{array} .\right.
$$


Ітераційна матриця системи така:

$$
\left\{\begin{array}{l}
x_{1}=\frac{3}{4}+\frac{1}{4} x_{2}-\frac{1}{12} x_{3} \\
x_{2}=\frac{8}{5}-\frac{1}{5} x_{1}+\frac{1}{5} x_{3} \\
x_{3}=\frac{8}{3}-\frac{1}{3} x_{1}+\frac{1}{3} x_{2}
\end{array} .\right.
$$

Ітераційний процес полягає у цикловому подаванні попередньо обчисленої величини на вхід обчислювача 3 певною трансформованою похибкою. Це означає, що функція переходів (функція перетворення) має залежати від кількості циклів ітерації $n$, тобто метрологічні стани ОК теж повинні залежати від $n$, що характерно для моделі автомата.

Похибка ітерації залежить від кількості ітерацій $n$. Отже, для цього обчислювального компонента регламентується динамічна похибка:

$$
\Delta_{i d}{ }^{(n)}=\Delta x_{i}{ }^{(n+1)-} \Delta x_{i}{ }^{(n)} .
$$

Подають на вхід обчислювального компонента масив значень початкових наближень:

$$
x_{1}{ }^{(0)}=x_{2}{ }^{(0)}=x_{3}{ }^{(0)} \text {. }
$$

Результати розрахунку наведено в табл. 8 .

\section{Табличя 8}

Розрахунок значень $\mathbf{x}_{i}$ залежно від кількості ітерацій

Table 8

Calculation of values $x_{i}$ depending on the number of iterations

\begin{tabular}{|c|c|c|c|c|c|c|}
\hline & \multicolumn{6}{|c|}{$\mathrm{n}$} \\
\hline $\mathrm{x}_{\mathrm{i}}{ }^{(\mathrm{n})}$ & 0 & 1 & 2 & 3 & 4 & 5 \\
\hline $\mathrm{x}_{1}{ }^{(\mathrm{n})}$ & 0 & 0,750 & 0,927 & 0,998 & 0,999 & 1,00 \\
\hline $\mathrm{x}_{2}{ }^{(\mathrm{n})}$ & 0 & 1,600 & 1,983 & 1,997 & 1,999 & 2,00 \\
\hline $\mathrm{x}_{3}{ }^{(\mathrm{n})}$ & 0 & 2,666 & 2,950 & 2.997 & 2,999 & 3,00 \\
\hline
\end{tabular}

Визначають значення динамічних похибок обчислень згідно з (9). Результати розрахунку наведено в табл. 9 і на рис. 1.

Таблиия 9

Визначення значень динамічної похибки ОК

Table 9

Determining the dynamic error values of computing component

\begin{tabular}{|c|c|c|c|c|c|}
\hline$\Delta \mathrm{x}_{i}{ }^{(\mathrm{n})}$ & 1 & 2 & 3 & 4 & 5 \\
\hline$\Delta \mathrm{x}_{1}{ }^{(\mathrm{n})}$ & 0,17 & 0,07 & 0,01 & 0,001 & 0,0 \\
\hline$\Delta \mathrm{x}_{2}{ }^{(\mathrm{n})}$ & 0,38 & 0,014 & 0,002 & 0,001 & 0,0 \\
\hline$\Delta \mathrm{x}_{3}{ }^{(\mathrm{n})}$ & 0,29 & 0,04 & 0,002 & 0,001 & 0,0 \\
\hline
\end{tabular}

$\Delta \mathrm{x}_{\mathrm{i}}$

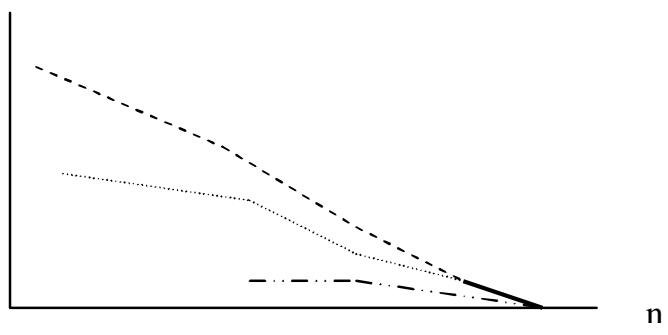

Рис. 1. Динамічна характеристика ОК, щуо реалізує ітераційну процедуру

Fig. 1. Dynamic characteristic of computing component implements the iterative procedure

За даними табл. 9 розраховуємо значення коефіцієнтів перетворення $f_{i}^{(n)}=\Delta \mathrm{x}_{i}^{(\mathrm{n}+1)} / \Delta \mathrm{x}$.

Табличя 10

Розрахунок значень коефіціснтів перетворення ОК

Table 10

Calculation of conversion coefficient values of computing component

\begin{tabular}{|c|c|c|c|c|}
\hline$f^{(\mathrm{n})}$ & 1 & 2 & 3 & 4 \\
\hline$f_{1}^{(\mathrm{n})}$ & 0,4 & 0,14 & 1,0 & 0,0 \\
\hline$f_{2}{ }^{(\mathrm{n})}$ & 0,11 & 0,14 & 0,5 & 0,0 \\
\hline$f_{3}{ }^{(\mathrm{n})}$ & 0,14 & 0,05 & 0,5 & 00 \\
\hline
\end{tabular}

За даними табл. 10 робимо висновок, що коефіцієнт перетворення (функція переходу) справді залежить від параметрів наближення. Отже, метрологічний стан ОК змінюється 3 кожним тактом ітераційного обчислення.

Визначання динамічної похибки обчислювального компонента, що реалізус алгоритм числового інтегрування методом Сімпсона. Обчислюють інтеграл виду

$$
I=\int_{0}^{1} \frac{1}{x+1} d x .
$$

За методом Сімпсона наближене значення інтеграла $I$ визначають за формулою:

$$
\begin{gathered}
I \approx h / 3\left[f\left(x_{0}\right)+4\left(f\left(x_{1}\right)+\cdot f\left(x_{n-1}\right)\right)+2\left(f\left(x_{2}\right)+\cdots\right.\right. \\
\left.\left.\cdots+f\left(x_{n-2}\right)\right)+f\left(x_{n}\right)\right],
\end{gathered}
$$

де $h$ - крок інтегрування, що визначають за формулою $h=(b-a) / n ; n-$ парна кількість елементів розбиття інтервалу інтегрування (параметр наближення); $f\left(x_{0}\right)$, $f\left(x_{N}\right)$ - значення підінтегральної функції на початку та в кінці інтервалу інтегрування відповідно; $f\left(x_{l}\right)$, $f\left(x_{2}\right) \ldots$ - поточні значення підінтегральної функції. 
Динамічну похибку визначають за формулою (8):

$$
\Delta_{y d}=y(n)-y_{d}
$$

де $y(n)$ - значення обчислюваної величини, отримане за фіксованого параметра наближення $n ; y_{d}$ - дійсне значення обчислюваної величини, одержане за умови збігу значень обчислюваної величини для двох послідовних значень параметра наближення.

Формується таблиця значень обчислювальної величини залежно від кількості елементів розбиття інтервалу інтегрування (табл. 11) Згідно 3 (8) розраховуються поточні значення похибки визначення інтеграла залежно від $n$ (динамічна похибка) (табл. 12). За даними табл. 12 розраховуємо значення функціональної залежності $f^{(n)}=\Delta \mathrm{y}^{(\mathrm{n}+1)} / \Delta \mathrm{y}^{(\mathrm{n})}$ (табл. 13).

\section{Таблицуя 11}

Залежність значень обчислювальної величини від кількості елементів розбиття інтервалу інтегрування

Table 11

The dependence of the calculated value of the number of integration interval elements

\begin{tabular}{|l|c|c|c|c|c|c|}
\hline$N$ & 2 & 4 & 6 & 10 & 12 & 14 \\
\hline$y(n)$ & 0,697 & 0,696 & 0,695 & 0,694 & 0,693 & 0,693 \\
\hline
\end{tabular}

Таблиия 12

Динамічна похибка ОК, що реалізус процедуру числового інтегрування

Table 12

Dynamic characteristic of computing component implements numerical integration procedure

\begin{tabular}{|l|c|c|c|c|c|c|}
\hline$n$ & 2 & 4 & 6 & 10 & 12 & 14 \\
\hline$\Delta y_{d}$ & 0,004 & 0,003 & 0,002 & 0,001 & 0,0 & 0,0 \\
\hline
\end{tabular}

Табличя 13

Значення коефіціснта перетворення ОК

Table 13

The value conversion coefficient of computing component

\begin{tabular}{|l|c|c|c|c|}
\hline$n$ & 2 & 4 & 6 & 10 \\
\hline$f^{(n)}$ & 0,75 & 0,66 & 0,5 & 0,00 \\
\hline
\end{tabular}

За даними табл. 13 робимо висновок, що коефіцієнт перетворення (функція переходу) ОК, який реалізує алгоритм числового інтегрування, залежить від параметрів наближення. Отже, метрологічний стан ОК змінюється залежно від кількості елементів розбиття інтервалів інтегрування (рис. 2).

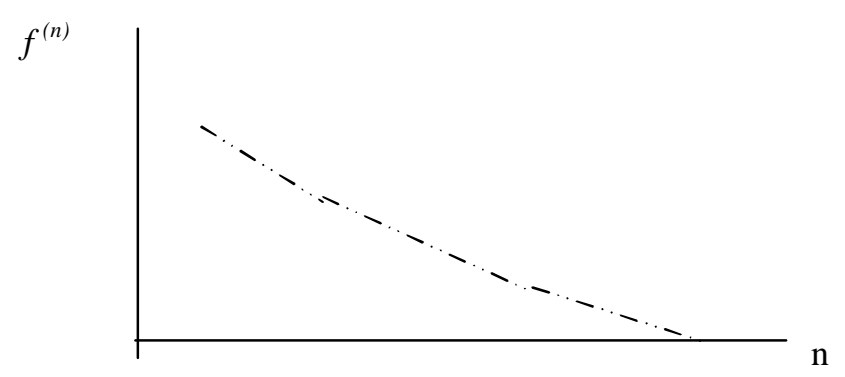

Рис 2. Динамічна характеристика ОК, щуо реалізує прочедуру числового інтегрування

Fig. 2. Dynamic characteristic of computing component implements the numerical integration procedure

Висновок. Динамічні властивості обчислювальних компонентів у представленні елементарних кінцевих автоматів переважно залежать від їхнього метрологічного стану в певний момент часу. Метрологічний стан ОК може бути незмінним, що характерно для елементарних автоматів Мілі, які реалізують обчислення за формулами, або змінюватися залежно від параметрів наближених обчислень - елементарні автомати Мура. Динамічні характеристики ОК мають детерміновані залежності від параметрів обчислень.

1. Кричевецьь О. М. // Праці IV Міжнародної науково-технічної конферениії “Метрологія - 2004”. Харків, 2004. 2. Кричевецьь О. М. // Международная конференция "Метрология и измерительная техника": сб. науч. Трудов. - T. VII - Харьков, 2005. 3. Кричевець О. // Вимірювальна техніка та метрологія. - 2008. - № 68. 4. Кричевеи А. М. // Матеріали міжнародної наукової конференції “Моделювання2010”: зб. наук. праць. - T. 2. - К., 2010 5. Кричевеиь О. М. // Украӥнський метрологічний журнал. 2014. - № 4. 6. Глушков В. М. Синтез циифровых автоматов. - М.: Физматгиз, 1962. 7. Кобринский Н. Е., Трахтенброт Б. А. Введение в теорию конечных автоматов. - М.: Физматгиз, 1962. 